\title{
Prácticas evaluativas en la clase de Educación Física: un asunto de desconocimiento \\ Evaluation practices in the Physical Education Class: An issue of lack of knowledge \\ *Carmen Rosa López Ávila, *William Orlando Arcila-Rodríguez, **Julián Eduardo Betancur Agudelo *Universidad de Caldas (Colombia), **Unidad Central del Valle del Cauca Uceva (Colombia)
}

\begin{abstract}
Resumen. Objetivo: el artículo se enfoca en la evaluación que se desarrolla en la clase de educación física teniendo en cuenta las técnicas e instrumentos que en dicho escenario se llevan a cabo. El proyecto se tituló: «Prácticas evaluativas en la clase de Educación Física en la ciudad de Manizales y Villamaría, Colombia» y tuvo como propósito develar las tendencias que se dan en los procesos formativos, a partir de la identificación e interpretación de estas. Metodología desarrollada: la investigación se asumió desde el paradigma cualitativo con enfoque complementario, en el cual se realizaron observaciones de clase, entrevistas a estudiantes y docentes de básica primaria, secundaria y media, las cuales fueron categorizadas mediante una codificación abierta, axial y selectiva. Resultados: se encontró que la evaluación en la clase de Educación Física tiende a ser comprendida de forma polisémica, es decir, dicho acto origina prácticas en las cuales se privilegie el desempeño físico o los aspectos actitudinales, que finalmente terminan opacando los contenidos conceptuales. Conclusiones y discusiones: es por esto, por lo que la evaluación se reduce meramente a una simple herramienta, la cual se enfoca a la verificación del aprendizaje y cumplimiento de tareas, desconociendo entonces la importancia que tienen la autoevaluación y los procesos metacognitivos.
\end{abstract}

Palabras Clave: Docente, Educación Física, Estudiante, Evaluación educativa, Prácticas pedagógicas.

Abstract. Objective: the article focuses on the evaluation that take place in the physical education class, considering the techniques and instruments that are carried out in this scenario. The project was titled: «Evaluative practices in the Physical Education class in the city of Manizales and Villamaría, Colombia» and its purpose was to unveil the tendencies that occur in the formative processes, based on the identification and interpretation of these. Methodology developed: the research was undertaken from the qualitative paradigm with a complementarity approach, in which class observations, interviews with students and teachers of elementary school, high school and middle school were conducted, which were categorized by means of an open, axial and selective coding. Results: it was found that the evaluation in the Physical Education class tends to be understood in a polysemic way, that is, this act originates practices in which physical performance and/or attitudinal aspects are privileged, which finally end up obscuring the conceptual contents. Conclusions and discussions: this is why evaluation is merely a simple tool, which focuses on the verification of learning and task accomplishment, thus ignoring the importance of self-evaluation and metacognitive processes.

Keywords: Teacher, Physical Education, Student, Educational evaluation, Pedagogical practices.

\section{Introducción}

Investigar sobre la evaluación en Educación Física (EF), permite precisar las dinámicas cotidianas consideradas naturales o raras en dicho contexto. Es por esto que, el interés de reconocer las formas en que el profesorado la desarrolla, se convierte en un asunto clave de todo proceso educativo. Sin embargo, poco se ha investigado sobre la evaluación desde un paradigma cualitativo (Atienza; Valencia \& Davís, 2018). De ahí, que el propósito general del estudio, se centra en develar las

Fecha recepción: 10-05-21. Fecha de aceptación: 06-10-21 Julián Eduardo Betancur Agudelo julianbeta333@gmail.com tendencias de las prácticas evaluativas, a partir de la identificación e interpretación de tendencias para posteriormente construir una propuesta de evaluación en la clase de educación física en la ciudad de Manizales y Villamaría.

Es así que, para abordar la evaluación en la EF, inicialmente hay que mencionar que es una de las áreas más apetecidas por los niños y niñas en el escenario escolar, en tanto se convierte en un espacio que permite mayor libertad de movimiento y a su vez de satisfacción y diversión (Zueck; Ramírez; Rodríguez \& Irigoyen, 2019), donde también tiene lugar la evaluación, pues a partir de esta se permite llenar de sentidos las acciones, pensamientos y representaciones de los diferentes actores. Por esto la clase de EF se ha convertido en 
escenario de investigación en el aspecto de la evaluación, en tanto, es un componente esencial en el proceso de enseñanza y aprendizaje que permite en primer lugar, visualizar los alcances de los estudiantes en su formación desde la esfera biopsicosocial, y que no sólo se puede reducir a un instrumento de calificación (Lucea, 2005; Ortegate, 2014; Rodríguez, \& Zulaika, 2016; Chaverra \& Hernández, 2019). En segundo lugar, por la preocupación que se da en la calidad de la educación del país, la cual constituye un elemento fundamental del máximo ente regulador de la educación cómo lo es el Ministerio de Educación Nacional para los procesos curriculares de las instituciones educativas y sus normativas (Decreto 1290, 2002).

Sin embargo, se ha evidenciado en la realidad educativa la falta de claridad en ¿Qué evaluar? ¿Cómo evaluar? y ¿Con qué evaluar? en dicha disciplina, situación evidente en el contexto de la investigación, en tanto, existen dinámicas que no muestran de manera precisa y acertada el desarrollo de un proceso rigurosos en torno a ella, dado que la tendencia actual de gran parte de los maestros está orientada a prácticas evaluativas desde «el dejar hacer»y «la observación» como lo muestran algunos estudios de referencia (Murcia \& Jaramillo, 2005; Betancur, López \& Arcila, 2018; Hortigüela, González \& Hernando, 2021); dando lugar a una observación sin criterios claros y poco fundamentados, en tanto, las dinámicas de las prácticas evaluativas se centran en el abordaje de unas dimensiones particulares. Adicional a ello, los instrumentos a tener en cuenta, se quedan en reducir el cuerpo a una máquina (esta visión entendida mediante la aplicación de test estandarizados) (Blázquez, 1990) y también, a buscar apoyo en técnicas sin un soporte que permita focalizar el proceso evaluativo en conductas observables sujetas a los propósitos de formación, desconociendo que la evaluación debe ser sistemática, intencional, que se centra en comprender las dinámicas que constituyen los procesos de enseñanza y aprendizaje, donde su sentido no puede ser la estandarización e instrumentalización del desempeño de los estudiantes, sino reconocer el potencial en el marco de sus particularidades, en las cuales están inmersas las diferentes dimensiones que configuran al ser humano; además de ser un apoyo para el docente en su proceso de reflexión y análisis, es indispensable involucrar asuntos como: estrategias didácticas, características del contexto, planes curriculares y prácticas pedagógicas.

En este sentido, como se observa en la Fig. 1., se propone asumir una postura evaluativa que supere las relaciones unidireccionales enfocadas a los procesos de calificación y comparación de pruebas estandarizadas, las cuales reducen la esencia misma de la naturaleza que configura al ser humano, en términos de Arcila \& Orrego (2014) sería «desarrollar sus prácticas, viciadas por un racionalismo técnico-instrumental, que ven el cuerpo como un conjunto de órganos, músculos, huesos y piel, que sirve como soporte al cerebro y albergue del conocimiento humano» (p. 16), por lo tanto, partir de la evaluación como mero instrumento de verificación es ver la formación de un ser cosificado, donde el estudiante también pasa a convertirse en un número, una cifra, desconociendo su complejidad.

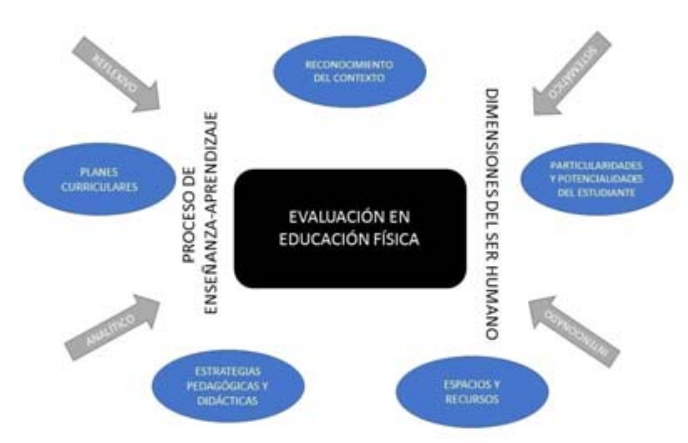

Figura 1. Aspectos de la evaluación. Elaboración propia.

Pensar la evaluación desde categorías como «aspectos evaluativos» $\mathrm{y}$ «técnicas e instrumentos», implica el conocimiento ante las dimensiones bio-psico-sociales, desde las cuales el docente de EF en el marco de sus intenciones formativas, debe definir de manera pertinente aquellas técnicas e instrumentos que se articulan a los propósitos que se pretenden desarrollar en sus clases, y con ello, promover los aprendizajes (López, 2014; Santos, 2014; Gallardo, López, Martínez y Carter, 2020; García, 2020).

Así nació este proyecto titulado «Prácticas Evaluativas en la Clase de EF» desarrollado en el marco del semillero: «Evaluación en EF» conformado por docentes universitarios y practicantes de la Universidad de Caldas pertenecientes al grupo de investigación «Mundos Simbólicos: Estudios en Educación y Vida Cotidiana».Y a su vez, con las diferentes inmersiones se pudo establecer y justificar la pertinencia del objeto de estudio, el cual está enfocado en la formación de los licenciados de la Licenciatura en EF, Recreación y Deporte.

\section{Metodología}

Inicialmente es necesario precisar la postura 
ontológica de la investigación, la cual se apoya en el construccionismo social (Castoriadis, 1983; Shotter, 1993; Tójar, 2006), en donde el interés estuvo enfocado en ver la dinámica de los acuerdos sociales establecidos por los actores que hacen parte de la clase de EF, con respecto a la evaluación y sus prácticas evaluativas, entendiendo que estas se configuran con base en las interacciones de los miembros de la comunidad educativa.

Debido a esto se decidió asumir un enfoque y diseño emergente, que posibilitó abordar el fenómeno desde sus particularidades y complejidades, teniendo presente que dicho objeto es quien da las pautas y las herramientas necesarias para la creación de una ruta que permita al investigador lograr los propósitos, sin perder de vista las exigencias que el proceso requería.

El enfoque introspectivo vivencial presentó gran importancia para el estudio, puesto que este se presenta como la interpretación de la realidad desde los simbolismos y el protagonismo por parte de los actores que la componen. Es así que, el investigador no se limita a concebir una realidad externa, por el contrario, es consciente que el conocimiento se constituye como la comprensión del mundo vivido (Padrón, 2001).

Teniendo en cuenta lo anterior, se asume la complementariedad (Murcia \& Jaramillo, 2008), como la posibilidad de comprender los hechos sociales desde su multidimensionalidad y complejidad que la determinan, desde posturas ontológicas, epistemológicas y metodológicas, reconociendo la inmanencia y trascendencia que dentro del objeto de estudio se configura así mismo, generar la posibilidad de indagar desde las realidades y las exigencias que el fenómeno estudiado requiere (Hurtado, Jaramillo, Zuñiga \& Montoya, 2005).

\section{Diseño Metodológico}

Se tuvieron en cuenta tres momentos, el primero de estos fue la preconfiguración de la realidad, donde se hizo un acercamiento a las instituciones educativas por parte de los investigadores, observando las diferentes clases de EF y encontrando unas precategorías que hicieron una pequeña interpretación de cómo se realizaban los procesos evaluativos dentro del área. Posteriormente, se generó un segundo momento denominado configuración de la realidad, en el cual se diseñaron unas entrevistas semiestructuradas basadas en las primeras observaciones y precategorías, para así, generar un primer proceso de saturación de la información sobre la evaluación. Por último, un tercer momento llamado reconfiguración de la realidad, se realizó una triangulación de la información, entre instrumentos y la teoría formal, lo que permitió una comprensión del fenómeno (Obsérvese en la Fig. 2.).

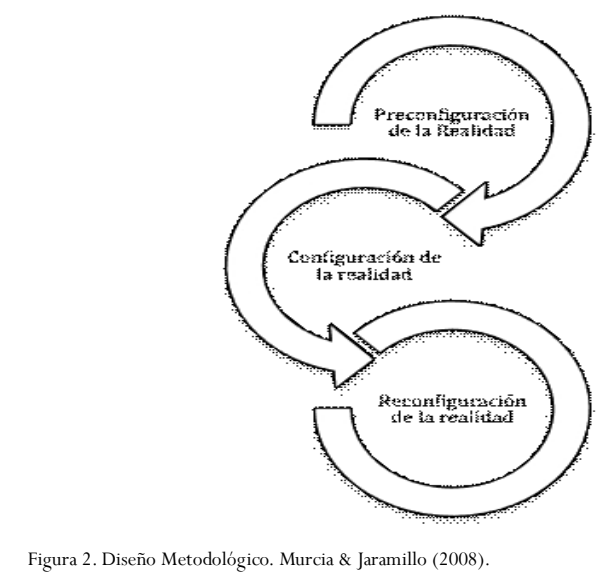

\section{Unidad de análisis y Unidad de trabajo}

Al momento de hacer el proceso de selección de los participantes de la investigación, se tuvo en cuenta como unidad de análisis las prácticas evaluativas en la clase de EF. Por otro lado, la unidad de trabajo estuvo conformada por 25 instituciones educativas de las ciudades de Manizales y Villamaría, 34 docentes titulares y 50 estudiantes. Los criterios de selección para las instituciones educativas estaban relacionados con un convenio marco de práctica educativa, para los docentes se tuvieron en cuenta a los profesores titulares de las diferentes instituciones que accedieron a participar con un consentimiento y asentimiento previo y los estudiantes fueron seleccionados desde las observaciones de clase en las que se evidenció su participación en las prácticas evaluativas.

Técnicas e instrumentos de recolección y procesamiento de la información

Para alcanzar este propósito la complementariedad posibilitó el uso de diferentes técnicas e instrumentos de investigación: Observación no participante (diario de campo), entrevista semiestructurada, revisión documental (Políticas y planes institucionales), grupos focales; los cuales fueron seleccionados teniendo en cuenta las necesidades que se requieren para lograr la comprensión del objeto de estudio y así validarlos socialmente con pruebas pilotos antes de su aplicación. Para la elaboración de estos instrumentos se tuvo en cuenta un primer momento de observación en dónde emergieron unas precategorías sobre las prácticas evaluativas, profundizadas en el desarrollo de las entrevistas y el análisis documental, permitiendo la indagación de con- 
cepciones y significados sobre la evaluación y el porqué del uso de ciertos instrumentos evaluativos.

El método de procesamiento fue el de codificación abierta, axial y selectiva propuestos por Strauss \& Corbin (2002), apoyados en el software Atlas. Ti $^{1}$, en donde se organizaron los datos recopilados en códigos y categorías, basados en los principios de comparación constante y saturación teórica permitiendo construir la red de relaciones y significados desde las representaciones que se dan en el contexto del fenómeno social. Con relación al método de análisis se realizó una triangulación de la información entre la teoría formal, la teoría sustantiva y la interpretación de los investigadores visualizando la dinámica de los acuerdos sociales.

\section{Resultados y discusión}

A continuación, se desarrollan las categorías emergentes denominadas: Técnicas e Instrumentos de Evaluación y Aspectos Evaluativos, acompañadas cada una de las subcategorías y su triangulación correspondiente. Es necesario reconocer las diferentes convenciones utilizadas para el procesamiento de la información las cuales brindan una mejor claridad de quiénes son los protagonistas en dicho relato, teniendo en cuenta el contexto en el que se desarrolla.

\begin{tabular}{ll}
\multicolumn{2}{c}{ Cuadro de Convenciones } \\
\hline E: & Entrevista \\
DC: & Diario de Campo \\
ES: & Estudiante \\
D: & Docente \\
IE: & Institución Educativa \\
\hline
\end{tabular}

\section{Categoría Técnicas e Instrumentos de Evalua- ción}

La presente categoría, evidenciada en la Fig. 3., muestra las dinámicas que existen con respecto a las diferentes técnicas e instrumentos que se desarrollan en las instituciones educativas de la ciudad de Manizales y Villamaría con respecto a la evaluación en la EF, donde los actores sociales (profesores y estudiantes) dieron cuenta de estos, evidenciados en los diferentes instrumentos de recolección.

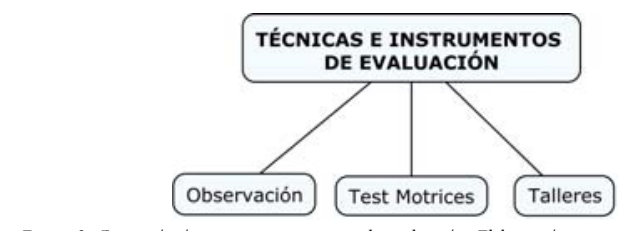

Esta categoría se refiere a las maneras en cómo es recolectada la información de las prácticas evaluativas con respecto a los procesos de enseñanza y aprendizaje llevados a cabo con los estudiantes en la clase, teniendo en cuenta aspectos prácticos, teóricos y actitudinales del estudiante, buscando así, una evaluación integral (Atienza, Valencia \& Devís, 2018).

Frente a esas técnicas e instrumentos se pueden encontrar las siguientes subcategorías como las más usadas por parte de los docentes, las cuales son la observación, talleres y test motrices.

\section{Subcategoría observación}

La observación es una de las técnicas más utilizadas por los docentes en el área de EF, ya que se utiliza como estrategia de seguimiento de los procesos realizados durante las clases por parte de los estudiantes, buscando evaluar el alcance o no de los propósitos y/o planteamientos de aprendizaje, tal y como lo manifiesta el siguiente docente:

"El plan evaluativo de la clase de EF se realiza a través de la observación de los procesos motrices de los niños y niñas de los grados de primaria, donde es fundamental retroalimentar conocimientos explicados y conocimientos ejecutados». (E11. D8. IE1).

Lo anterior muestra cómo esta técnica, sólo se enfoca en una de las dimensiones de evaluación específicamente en el ámbito procedimental, dejando a un lado la parte conceptual y actitudinal; siendo la evaluación un proceso de repetición de movimientos donde el alumno desconoce el por qué y para qué de su ejecución, se preocupa por cumplir con una serie de tareas motrices de forma individual que lo cuantifique dentro de una escala de valoración, impidiendo así, procesos de interacción grupal y reconocimiento de ideas previas ante cada temática. Frente a esto, algunos autores expresan que la evaluación por observación es un procedimiento que se basa en la percepción del docente, la cual debe contar con el apoyo de otras técnicas y la retroalimentación que permitan tener mayor precisión (González \& Lleixas, 2010; Barrientos, López \& Pérez, 2019).

En este sentido, se resalta la importancia de realizar procesos observacionales que cuenten con instrumentos pertinentes que eviten sesgos en la evaluación de los aprendizajes del estudiante, puesto que esta técnica pierde objetividad cuando se relaciona sólo con aspectos volitivos (Hortiguela, Gonzalez \& Hernando, 2021; Herrero, Manrique, \& López, 2021).

Cuando el docente plantea una evaluación integral, puede recurrir a la observación reconociendo los ejes evaluativos conceptual, procedimental y actitudinal, por medio de un proceso continuo de evaluación, donde 
apoyado por la observación directa de los estudiantes, busca contribuir a mejorar en cada uno de ellos aquellos planteamientos curriculares que se proponen para la clase de EF.

"Es integral, es continua se evalúa al estudiante desde el inicio hasta el final, se observan acciones y conductas de trabajo en equipo. Con los logros que plantea la institución educativa, con observación directa de los estudiantes, a través de la resolución de talleres y la disposición de los estudiantes» (E1. D7. IE3).

Desde las directrices MinEducación, en el decreto 1290 del 2010 (artículo 3 propósitos de la evaluación institucional de los estudiantes) se establece «Proporcionar información básica para consolidar o reorientar los procesos educativos relacionados con el desarrollo integral del estudiante» (pp.1). Además, se enmarca como un derecho de los estudiantes en su artículo 12, «Ser evaluado de manera integral en todos los aspectos académicos, personales y sociales» (pp.4). Por lo cual, es un deber tanto de la institución como del docente velar por el compromiso y rigurosidad de los procesos evaluativos de las distintas áreas.

En la información recopilada, también se evidencian docentes que toman por decisión propia el uso de la observación como técnica de evaluación en la clase, construyendo criterios autónomos que evalúan el comportamiento y desempeño motriz de cada estudiante. Tal cómo se evidencia en el siguiente relato:

"En el momento de la clase, pues es difícil para mí porque no tengo el tiempo para sacar procesos de evaluación, a través de la observación durante toda la clase yo estoy interpretando el comportamiento de los chicos a nivel motriz» (E4. D8. IE7).

Lo anterior, muestra desconocimiento por parte del profesor acerca de la evaluación, dado que indica que la observación no hace parte de esta, promoviendo así la valoración de comportamientos y conductas motrices de los estudiantes cómo criterio de calificación. En este sentido, Hamondi, López \& López (2015) expresan que la sistematización de la producción del estudiante, acompañada del juicio de valor la constituye.

Por lo tanto, la observación como única forma de abordar la evaluación no permite interpretar las diferentes acciones motrices realizadas por los estudiantes, desde diferentes perspectivas asociadas a la interacción dialógica, apoyo y superación de dificultades que los alumnos presentan en las temáticas desarrolladas; el profesor entonces, solo observa el desempeño motriz del estudiante, y según su propio criterio establece parámetros de los aprendizajes en la ejecución de las actividades realizadas, desconociendo la normatividad establecida y las particularidades de los estudiantes.

Como menciona Buscà, et. Al. (2010 pp. 265), «los procedimientos de evaluación basados en la observación, constatamos que en la mayoría de casos se emplean listas de control con la intención de verificar la participación y la presencia del estudiante durante el proceso de enseñanza y aprendizaje», se puede encontrar entonces un problema en el docente al momento de evaluar, pues acompañado de la observación solo se encuentra la heteroevaluación hacia el estudiante a nivel motriz, desconociéndose a los demás actores participantes en la evaluación. Adicional a ello, basado en su propia experiencia el docente construye una calificación o puntuación en una escala determinada; como lo expresa el autor el docente puede implementar listas de control para facilitar su proceso de observación, y así mejorar sus criterios de evaluación siendo estos planeados con anterioridad para cada tema en específico y eje evaluativo.

Finalmente, se puede evidenciar que la observación es la técnica más empleada tanto por los docentes y referidas en los planteamientos institucionales, aunque en algunos casos su aplicación es insuficiente, puesto que se limitan al denominado «ojímetro ${ }^{2} »$, y es por esto por lo que debe complementarse con otras técnicas para posibilitar una evaluación integral.

\section{Subcategoría talleres}

La categoría talleres, surge de la información encontrada en los acuerdos sociales de la clase de EF, como una de las técnicas que los docentes emplean para evaluar el proceso de aprendizaje de los estudiantes, enfocada en el componente conceptual a través de exámenes, consultas, exposiciones y talleres.

De acuerdo con Reyes (2000), esta técnica permite articular la teoría y práctica, con la finalidad de tener una realidad amplia a partir de la reflexión y comunicación con los agentes participantes, siguiendo a Tamayo, Niño, Cardozo \& Bejarano (2017), se promueve la construcción de conocimiento, la participación democrática y la reflexión sobre las experiencias. En la realidad de las prácticas evaluativas, los docentes llevan a cabo en alta medida la implementación de talleres, en tanto, permiten generar un ambiente que integra principalmente dos ejes (procedimental, actitudinal), sin embargo, el docente desconoce que, a partir de esta técnica, se puede llevar a cabo un proceso integral, donde se condensan las tres dimensiones, siempre y cuando el 
docente logre focalizar los intereses de abordar cada una de ellas mediante el taller.

"[...] donde se tienen en cuenta todos los procesos vistos en clase, que se traduce al sistema en un formato cualitativo $y$ cuantitativo para observar procesos educativos, donde se determina avances $y$ aprendizajes a través de planteamientos para la clase como talleres, actividades lúdicas, trabajos en clase, trabajos cooperativos...» (E11. D4. IE6).

Pero esta práctica se ha limitado como una alternativa que implementan algunos docentes para los estudiantes que no tienen la posibilidad de participar de la clase, ya sea por alguna incapacidad médica o por un mal comportamiento, lo que deja en duda los propósitos y finalidades de estas.

En síntesis, se debe entender el taller como aquella posibilidad integradora en la cual se logra relacionar la realidad a partir de discusiones y situaciones prácticas apoyadas en sustentos teóricos constituyéndose en la generación de conocimiento. Tal como lo manifiesta Maya, (2007) quien considera que esta estrategia permite el desarrollo de competencias desde el aprender a ser, el aprender a aprender y el aprender a hacer, todo ello apuntando a la formación integral.

\section{Subcategoría Test Motrices}

Los test motrices hacen referencia a las diferentes pruebas físicas estandarizadas que se encargan de medir ciertas aptitudes y condiciones físicas de los estudiantes, lo cual finalmente se representa a través de un dato cuantitativo.

De acuerdo con Baena, Granero \& Ruiz (2010), a través de la aplicación de los test motrices, se pueden conocer aquellos estudiantes con problemas cognitivos, coordinativos y afectivos, para así conocer su estado de evolución, para diagnosticar e iniciar un profundo control de los estudiantes y mejorar sus capacidades. En relación con lo anterior, se puede observar que algunas prácticas evaluativas en la clase de EF están orientadas a la valoración de la condición física, este tipo de evaluación ha tenido gran aceptación entre los docentes, dado que, año tras año han dado uso de test para evaluar al estudiante, siendo uno de los instrumentos con mayor recurrencia en las prácticas evaluativas. Tal como se menciona en los siguientes relatos:

¿Cómo los evalúan en la clase de EF?

Con ese test de Cooper y el físico que uno tenga (E2. ES5. IE7) $[\ldots]$.

[...] Trabajó resistencia, donde dividió el grupo en dos subgrupos el primer grupo trabajó salto a la cuerda en distintas formas (A pie junto, separado, alternando pie derecho e izquier- do) intervalos de un 1.30 segundos y el otro grupo subía y baja gradas con un tiempo de 3 minutos continuos y 1 de descanso los dos subgrupos [...] (DC1. IE14)

En lo anterior, se evidencia el reconocimiento por parte de los estudiantes del uso de test y pruebas estandarizadas como estrategia evaluativa del docente, por lo tanto los test como técnica experimental asumen un papel relevante a la hora de conocer el desarrollo motriz, antropométrico y físico, permitiendo una reorientación de los procesos formativos; sin embargo, en las observaciones logradas en la realidad escolar, se presentan espacios en los cuales son utilizados los diferentes test desde una perspectiva más instrumental y como requisito de aplicación desde los planes curriculares institucionales, perdiendo de vista el análisis y reflexión de las características y fundamentos pedagógicos que los constituyen, al igual que la verdadera esencia y finalidad de esta herramientas a la hora de evaluar la población escolar.

Frente a lo propuesto por Ruiz (2009 pp. 5), al referirse a los test motrices como «prueba definida que implica la realización por parte de todos los sujetos examinados de una tarea idéntica, con una técnica precisa, para la apreciación del éxito y del fracaso o para la expresión numérica del grado de logro», se observa que este procedimiento centra su interés en el rendimiento motriz, por lo tanto, se convierte en una herramienta importante en el área, dado que permite identificar el estado antropométrico (Palma, et. Al, 2020) y su aptitud física (Dávila, et. Al, 2020), como punto de partida para resignificar las tareas motrices y los cambios a nivel físico.

Pese a que es un procedimiento propio de área, no se puede asumir como la única opción de instrumento de evaluación, dado que sólo se interesa por la dimensión motriz, perdiendo de vista la integralidad requerida de la evaluación en el escenario escolar donde convergen aspectos actitudinales, sociales y emocionales, además que dichas pruebas se focalizan en las observaciones y análisis exclusivos del docente.

[...] Hay muchas formas de evaluar en la clase EF primero pues en las clases de EF se evalúa mediante pruebas, se valora mediante test (E5. D5. IE7).

En síntesis, los intereses evaluativos por parte del docente se ubican desde la capacidad física que logran desarrollar los estudiantes a través de los proceso de formación orientados, sin embargo, el aspecto actitudinal juega un papel relevante a la hora de construir criterios y desarrollar procesos de calificación; y a pesar de los avances teóricos que emergen en el campo de la eva- 
luación del área, existe poca reflexión pedagógica ante la evaluación, puesto que se pueden encontrar algunas técnicas e instrumentos mencionadas en la teoría formal, que no tienen ninguna aplicación por parte de los docentes, esto conllevo a generar cuestionamientos cómo ¿podría ser desconocimiento de ellas?, ¿acaso son muy complejas para su aplicación?, posiblemente, por estas y otras razones no son utilizadas. Por lo cual, se evidencia una mirada reduccionista de la evaluación en las prácticas docentes propias de las lógicas experimentales de la investigación, que no vislumbran el pensamiento complejo en el desarrollo del aprendizaje de la educación física.

\section{Categoría Aspectos Evaluativos}

En la presente categoría (véase en la Fig. 4), se logra evidenciar diferentes aspectos que son asumidos por docentes y estudiantes en la clase de EF durante las prácticas evaluativas. Entre ellos se resaltan los siguientes componentes:

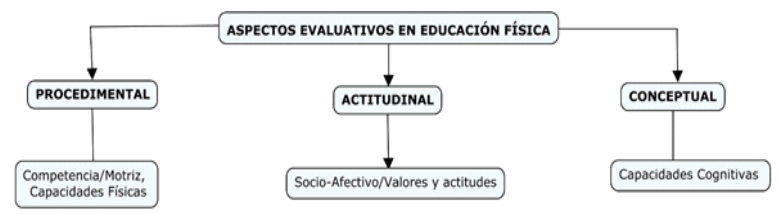

Figura 4. Aspectos Evaluativos en EF. Elaboración propia.

\section{Subcategoría Procedimental}

El aspecto procedimental hace alusión al interés del docente en centrar la atención en las acciones motrices (hacer) que llevan a cabo los estudiantes en la clase de EF en términos de rendimiento y participación

«Pues el profesor nos lleva a los diferentes escenarios deportivos, nos hace los diferentes test y todo eso, y esas son como las formas más comunes» (E.12. ES.8. IE. 11)

Lo planteado anteriormente, muestra cómo las prácticas evaluativas se centran en elementos de orden práctico, enfocados principalmente al rendimiento, resultados físicos y motrices (Rodríguez et al, 2017), tratando con ello de buscar la comprobación en relación con los objetivos formativos planteados, los cuales están enmarcados en aprendizajes de orden procedimental, tal como se expone en la gráfica 4 (Aspectos Evaluativos en $E F)$.

Frente a estas dinámicas, se observa un proceso en el cual el docente a partir de su paradigma instituido en lo biomédico e higiénico de la EF desde el siglo XIX, genera prácticas centradas en una perspectiva dualista del cuerpo, la cual es proyectada en sus prácticas evaluativas, es decir las finalidades del proceso de ense- ñanza y aprendizaje se orientan al individualismo y desarrollo del organismo, dejando a un lado la colectividad, entendiendo el cuerpo como un todo configurado por distintas dimensiones. Por lo tanto, su objetivo está centrado en el cultivo del cuerpo, para con ello lograr una buena salud gracias al desarrollo de cualidades físicas, destrezas, fuerza, disciplina y seguridad, aspectos que permitirán lograr un cuerpo recio para afrontar situaciones de la vida cotidiana.

Según Camacho (2003), la existencia de dos ópticas como la higiénica y el cuerpo como instrumento productivo, reflejan la preocupación de los docentes en desarrollar instrumentos evaluativos que permitan valorar el rendimiento motriz y el desarrollo de capacidades físicas y destrezas motrices. De manera que, frente a las posturas e ideologías que tienen los docentes se observa esa escisión entre cuerpo- mente, dejando de lado otros aspectos que requieren de igual forma la importancia de ser trabajados y valorados en la clase; de acuerdo con lo anterior, los proceso evaluativos implementados en la clase de EF se ven influenciados hacia promover una lógica del Derby, es decir, fomentar procesos competitivos donde prima el más fuerte y el mejor rendimiento, resultado de los productos que cada estudiante obtiene desde el campo motriz y habilidades físicas, ubicando así el cuerpo en un rango de jerarquización, clasificación y estratificación; en términos de Portela (2006, pp. 74), «Se trata de una institución que decide sobre el cuerpo, lo organiza, jerarquiza y lo pone a funcionar de acuerdo a una intencionalidad institucionalizada.»

\section{Subcategoría actitudinal}

La presente subcategoría se refiere a las actitudes que son valoradas por el docente frente a las interacciones que se generan en el desarrollo de la clase de EF, correspondiendo estas a la participación en la clase (querer hacer), el fortalecimiento de los valores, el cumplimiento de las normas y la presentación personal.

En lo encontrado, se observa que algunos docentes privilegian el aspecto actitudinal sobre los demás componentes e inclusive los opacan en su totalidad, en tanto valoran la disposición como elemento fundamental del proceso de enseñanza y aprendizaje, ignorando algunos de los fines que desde la $\mathrm{EF}^{3}$ se pretenden desarrollar.

Para mí lo más importante es la motivación del estudiante, a mí no me importa si es el más "ducho» o es el más amotriz, si viene con ganas de aprender y se encariña con el cuento, entonces conmigo le va a ir bien. (E.1.D.5 .IE.1) 
Si bien, es importante reconocer el aspecto comportamental en el proceso de enseñanza y aprendizaje, en tanto la disposición por parte del estudiante y la generación de un clima apropiado para la orientación de las clases y el cumplimiento de los planteamientos institucionales (Manual de convivencia y el currículo formal); no se puede olvidar que existen objetivos propios del área que involucran aprendizajes que superan la buena intención o el esfuerzo de realización o no de los ejercicios propuestos.

[...] tratamos de evaluar el esfuerzo que el estudiante hace durante la clase, la actitud, la responsabilidad, la disposición, el ánimo que uno le ve en la clase, y a partir de ahí uno ya evalúa el proceso de una forma subjetiva, de acuerdo pues a esas características, por ejemplo, si el estudiante se vuelve muy perezoso, o uno lo ve es que no quiere hacer la clase sino que lo ve esforzándose [...] de todas maneras se les valorará ese esfuerzo y esa actitud.(E.7.D.2.IE.5)

Es importante destacar la complejidad que implica para los docentes la evaluación de este componente, en tanto la rigurosidad que se tiene es reducida a lo subjetivo a partir de la observación de diversas conductas que cuentan con un criterio impuesto por el docente, dejando a un lado la postura que asume el estudiante frente a las actitudes asumidas en clase, convirtiéndose en un actor pasivo de su proceso evaluativo.

El docente debe desarrollar procesos donde se vean implícitos aquellos valores que se pretenden fortalecer a partir de sus prácticas pedagógicas, didácticas y metodológicas, por lo tanto, el objeto de valoración debe estar centrado en los propósitos formativos que en el campo actitudinal se van a desarrollar, además debe estar acompañadas por el estudiante (Orrego \& Portela, 2009).

Significa entonces, que las prácticas evaluativas desarrolladas en la clase de EF se orientan hacia los ejes conceptual, procedimental y actitudinal: el conceptual hace referencia a consultas, exámenes, exposiciones y talleres, acciones desarrolladas por los estudiantes para obtener una nota; el componente procedimental, se refiere a la ejecución de acciones motrices y exposiciones, que se desarrollan en toda una clase o todo un periodo académico; el componente actitudinal, hace alusión a la participación e interacción con el otro y lo otro, a partir de la disciplina, responsabilidad, respeto, tolerancia y buen comportamiento durante la clase de EF.

\section{Subcategoría conceptual}

Hace referencia a elementos de orden teórico que se desarrollan en la clase de EF de forma oral y escrita. De manera que, hay una estrecha relación con una de las subcategorías mencionadas anteriormente (talleres), en tanto este instrumento se convierte en un medio de evaluación del aspecto cognitivo hacia el estudiante.

Cuando nos ponen teoría simplemente es en una hoja. Nos sentamos y empezamos a desarrollarlo (E.9. ES.5. IE.12)

Es importante mencionar que el componente conceptual se desarrolla especialmente en espacios o escenarios cerrados como el salón de clases, por lo tanto, se presenta una situación de fragmentación de cada uno de los componentes involucrados en el proceso de enseñanza y aprendizaje (procedimental, actitudinal y conceptual) desconociéndose el carácter integral que se debe llevar a cabo.

[...] si es en el salón, en cuaderno o en una hoja (E.6. ES.10.IE.10)

Esta situación en primer lugar deja evidente que en ocasiones los docentes se limitan a la memorización de datos por parte de sus estudiantes, como lo asume Castillo (2002), al referirse a la evaluación desde una perspectiva estática, la cual consiste en asimilar datos simples, sin lograr la consolidación y comprensión de conceptos, principios y teorías.

El dato es un conocimiento fragmentado, parcial y concreto. El concepto, por el contrario, implica mayor relación entre varios hechos o datos, mayor abstracción y más capacidad explicativa.(Castillo, 2002 pp. 59)

En la realidad de las prácticas evaluativas en el campo de la EF, se presenta una tendencia que se proyecta hacia ver el aspecto conceptual como un requisito que se aborda en la clase, pero, que no tiene mayor predominio, pues el aspecto que tiene mayor relevancia son las acciones motrices que desarrolla el estudiante; Sin embargo, cuando se hace uso de algunas estrategias evaluativas, en ocasiones significa un castigo para el estudiante, debido a la fuerte tendencia de fomentar el aspecto motriz en la clase de EF (Rodríguez \& Zulaika, 2016).

Lo anterior, refleja la ausencia de una evaluación formativa, pues una de sus características es la integralidad, así como lo menciona Camacho (2003 pp. 152) «[...] al evaluar, deben tenerse en cuenta todos los factores o componentes que intervienen de manera directa o indirecta en el desarrollo del área de EF, recreación y deporte» que permitan el perfeccionamiento de los procesos de enseñanza y aprendizaje, tanto desde la perspectiva del estudiante como del docente (Barrientos, et al., 2019); Por lo tanto, se dejan de lado cuestiones 
relacionadas con la posibilidad de transformación que brinda la evaluación desde lo cognitivo y actitudinal, pues los intereses priman hacia aspectos motrices y focalizada solo en el estudiante, perdiendo de vista la evaluación que le apueste al aprendizaje dialógico (López et al., 2019).

Al respecto, Sicilia et. Al (2006), coinciden con los elementos encontrados en el presente estudio, tal y cómo se refleja en la siguiente conclusión en donde el profesorado de la básica primaria prioriza los aprendizajes actitudinales (46.35\%), mientras que en la secundaria se centra la atención en aprendizajes procedimentales (43.71\%) dejando en un segundo plano el componente conceptual.

\section{Conclusiones y reflexiones}

En los acuerdos sociales encontrados en las prácticas evaluativas en la clase de EF se puede decir que:

Los docentes del área desarrollan sus prácticas evaluativas en el marco de sus creencias y paradigmas ignorando los avances epistemológicos en el área, es decir, si se asume una mirada fisicalista de la EF llevan a cabo procesos orientados al rendimiento físico-motriz del estudiante, estandarizando e instrumentalizando la formación integral del estudiante y opacando otros elementos propuestos en los planes curriculares y orientaciones pedagógicas.

El componente de mayor interés en la evaluación se ubica en el campo actitudinal, siendo reducida al criterio subjetivo del docente, lo cual subordina aspectos fundamentales en el aprendizaje del área disciplinar como lo son el procedimental y el conceptual, para así responder al deber ser de la evaluación en el escenario educativo.

La educación de hoy no puede ser la misma de ayer, ni la misma de mañana, de manera que se hace necesario llevar a cabo un trabajo reflexivo para atender a las dinámicas de las nuevas generaciones. Por lo tanto, en lo que respecta a técnicas e instrumentos evaluativos, es necesario responder a las particularidades de los estudiantes a partir de la configuración de estrategias evaluativas que son implementadas en el escenario escolar, esto implica pensar la evaluación desde y para los estudiantes como aspecto sistemático, reflexivo e intencional, para comprender el proceso de enseñanza y aprendizaje y superar la mirada estandarizada e instrumental del desempeño de los estudiantes.

Aunque muchos de los docentes y estudiantes manifiestan que existen procesos autoevaluativos y coevaluativos, se puede concluir que es necesario llevar a cabo procesos metacognitivos dentro del aula (Panadero \& Tapía, 2014), para así, poder generar transformación en los aprendizajes de los estudiantes y la clase de EF.

Pese a que existen diversos estudios en el campo de la evaluación en EF, los cuales concluyen que las dificultades en las prácticas son múltiples. Se hace necesario repensar los procesos que se vienen desarrollando en la formación de los futuros licenciados de la disciplina, a través de procesos investigativos de orden comprensivo y crítico sociales, que permitan configurar nuevas prácticas evaluativas que atiendan a la naturaleza compleja del ser humano y del área.

\section{Referencias}

Arcila,W., Orrego, F. (2014). La educación física en la actualidad: apuestas por una nueva concepción. Revista Impetus. Vol. 8 pag. 25-33 Universidad de los llanos. Colombia.

Atienza, R., Valencia, A., \& Devís, J. (2018). Experiencias de Evaluación en Educación Física. Una Aproximación desde la Formación Inicial del Profesorado. Estudios Pedagógicos, 44(2), 127-147. doi:10.4067/ S0718-07052018000200127

Baena, A., Granero, A., Ruiz, P. (2010). Procedimientos e instrumentos para la medición y evaluación en el sistema educativo. Journal of Sport and Health Research, Vol. 2(2). P.63-76 Universidad de Murcia. España.

Barrientos, E., López, V., \& Pérez, D. (2019). ¿Por qué hago evaluación formativa y compartida y/o evaluación para el aprendizaje en EF? La influencia de la formación inicial y permanente del profesorado, Retos, Vol 36, p. 37-43. https: / /doi.org/10.47197/retos.v36i36.66478

Betancur-Agudelo, J.E., López-Ávila, C.R. y Arcila-Rodríguez, W.O. (2018). El docente de Educación Física y sus prácticas pedagógicas. Revista Latinoamericana de Estudios Educativos. 14 (1), pág. 15-32 DOI: $10.17151 /$ rlee.2018.14.1.2

Blázquez, D. (1990). Evaluar en Educación Física. Editorial INDE Barcelona España.

Buscà, F., Pintor, P., Martínez, L., y Peire, T. (2010). Sistemas y procedimientos de evaluación formativa en docencia universitaria: Resultados de 34 casos aplicados durante el curso académico 2007-2008. Revista estudios sobre educación vol. 18 p. 255-276

Camacho, H. (2003). Pedagogía y Didáctica de la Educación Física. Editorial kinesis

Camacho, H; Marcano, N. (2003). El Enfoque de Investigación Introspectivo Vivencial y sus Secuencias Operativas. Algunos Casos de Estudio. Omnia.

Castillo, S. (2002). Compromisos de la Evaluación Educativa. Editorial Prentice Hall. España

Castoriadis, C. (1983). La institución imaginaria de la sociedad.Vol. 1. Marxismo y teoría revolucionaria. Barcelona: Tus Quest Editores CEDE, 63.28.

Chaverra, F. y Hernández, J. (2019). La planificación de la evaluación en educación física: Estudio de casos sobre un proceso desatendido en la enseñanza. Revista Electrónica Educare, 23(1),1-21.[fecha de Consulta 21 de Julio de 2020]. ISSN: Recuperado de: https: / / www.redalyc.org/articulo.oa?id=1941/194159163012

Dávila Grisalez, A., Mazuera Quiceno, C., Carreño Herrera, A., \& Henao Corrales, J. (2020). Efecto de un programa de entrenamiento interválico aeróbico de alta intensidad en población escolar femenina con sobrepeso 
u obesidad (Effect of a high intensity aerobic interval training program on an overweight or obese female school population). Retos, 39, 453458. https: / / doi.org/10.47197/retos.v0i39.78200

Gallardo F., LópezV., Martínez, C., y Carter B. (2020). Evaluación formativa en educación física y atención a la diversidad. Magis, Revista Internacional De Investigación En Educación, 12(25), 169-186. Recuperado de: https://doi.org/10.11144/Javeriana.m12-25.efef

García, L. (2020). La evaluación en Educación Física Escolar. Editorial Kinesis

Hamodi, C.; López, V.; López, A. (2015). Perfiles educativos. Medios, técnicas e instrumentos de evaluación formativa y compartida del aprendizaje en educación superior. Vol. (37), 146-161. México.

Herrero-González, D., Manrique Arribas, J. C., \& López-Pastor, V. (2021). Incidencia de la Formación Inicial y Permanente del Profesorado en la aplicación de la Evaluación Formativa y Compartida en Educación Física (Incidence of Pre-service and In-service Teacher Education in the application of Formative and Shared Assessment. Retos, 41, 533 543. https: / / doi.org/10.47197/retos.v0i41.86090

Hortiguela, D., Gonzalez, S., Hernando, A. (2021). Do we really assess learning in physical education? Teachers' perceptions at different educational stages, Retos, 42, 643-654. https: / /doi.org/10.47197/ retos.v42i0.88686

Hurtado, D., Jaramillo, L., Zúñiga, C. y Montoya, H. (2005). El imaginario del joven ante la clase de educación física. Popayán: Universidad del Cauca.

LópezV., Molina, M., Pascual, C., \& Manrique, J. (2019). La importancia de utilizar la Evaluación Formativa y Compartida en la Formación Inicial del Profesorado de Educación Física: los Proyectos de Aprendizaje Tutorado como ejemplo de buena práctica, Retos, Vol. 37, p.620627. https: / / doi.org/10.47197/retos.v37i37.74193

López, A. (2014). La evaluación como herramienta para el aprendizaje. Conceptos, estrategias y recomendaciones. Editorial Magisterio.

Lucea, J. (2005). Evaluación Formativa como Instrumento de Aprendizaje en educación fisica. Barcelona España: INDE.

Maya, A. (2007). El taller educativo ¿Qué es? fundamentos, cómo organizarlo y dirigirlo y cómo evaluarlo.

Ministerio de Educación Nacional. (2009). Decreto 1290 por el cual se reglamenta la evaluación del aprendizaje y promoción de los estudiantes de los niveles de educación básica y media.

Ministerio de Educación Nacional. (2010). Documento orientaciones pedagógicas para la Educación Física, Recreación y Deporte.

Murcia, N., Jaramillo, L. (2005). Imaginarios del joven colombiano ante la clase de educación física. Revista Latinoamericana de Ciencias Sociales, Niñez y Juventud, 3(2), 175-212. Retrieved August 02, 2017. Recuperado de: h t t p : / / w w w. s c i e lo. o r g. c o / scielo.php?script $=$ sci_arttext\&pid=S1692715X2005000200007\&lng $=$ en\&tlng $=$ es.

Murcia, N., Jaramillo, L. (2008). Investigación cualitativa:La complementariedad. Segunda edición. Armenia. Editorial Kinesis.

Orrego, J. F., Portela, H. (2009). Sentido de la alteridad en la formación del licenciado en Educación Física. Revista Educación y Deporte, n. 282, 49-57.

Ortegate, G. (2014). Evaluación en educación física: concepciones y prácticas docentes. Voces y Silencios: Revista Latinoamericana de Educación,Vol. 5 (2). 99-117).

Padrón, J. (2001) La estructura de los procesos de investigación. Revista Educación y Ciencias Humanas. Vol VII. $N^{\circ} 15$. 7-12. Universidad Nacional Experimental Simón Rodríguez.

Palma Pulido, L., Méndez Díaz, C., Cespedes Manrrique, A., Castro Mejía, J., Viveros Restrepo, A., Garzón, K. A., Arroyave Aristizabal, A., Cardona,W., \& Restrepo Ríos, Álvaro. (2020). Asociación entre la composición corporal y la condición física en estudiantes de grado sexto, pertenecientes a la institución educativa moderna de Tuluá,
Colombia año 2019 (Association between body composition and the physical condition in sixth grade st. Retos, 39, 539-546. https:// doi.org/10.47197/retos.v0i39.77988

Panadero, E. y Tapia, J. (2014). Teorías de autorregulación educativa: Una comparación y reflexión teórica. Psicología educativa (20) 11-22. DOI: http://dx.doi.org/10.1016/j.pse.2014.05.002

Portela, H. (2006). Los conceptos en la educación fisica. Editorial kinesis. Colombia

Reyes, M. (2000). «El taller en el trabajo social. Taller de integración de teoría y práctica. Editorial humanidades. Buenos Aires. Argentina.

Rodríguez, J., Curilem, C., Berral de la Rosa, F. \& Almagià, A. (2017). Evaluación de la educación física escolar en enseñanza secundaria. RETOS. Nuevas Tendencias en Educación Física, Deporte y Recreación, (31), 76-81. Disponible en: https://www.redalyc.org/ articulo. oa?id $=345750049014$

Rodríguez, J., Zulaika, L. (2016). Evaluación en educación física. Análisis comparativo entre la teoría oficial y la praxis cotidiana. Sportis. Revista Técnico-científica del Deporte Escolar, Educación Física y Psicomotricidad. Vol II, n. 3; p 421-438 Coruña, España

Santos G. (2014). La evaluación como aprendizaje «Cuando la Flecha impacta la diana». Madrid: Narcea.

Shotter, J. (1993). Realidades Conversacionales. Buenos Aires: Amorrortu Editores.

Sicilia, A.; Delgado, M.; Sáenz, L.; Manzano, J.; Varela, R.; Cañadas, J.; Gutiérrez, M. (2006). La evaluación de aprendizajes en educación física. Diferencias en función del nivel educativo. European Journal of Human Movement, vol. 17, pp. 71-93 Asociación Española de Ciencias del Deporte Cáceres, España Strauss, A., \& Corbin, J. (2002). Bases de la Investigación Cualitativa: Técnicas y Procedimientos para Desarrollar la Teoría Fundamentada. Medellín: Contus, Universidad de Antioquia.

Tamayo, A. Niño, Z. Cardozo, L. y Bejarano, O. (2017). ¿Hacia dónde va la evaluación? Bogotá: Investigación IDEP.

Tójar, J. (2006). Investigación Cualitativa Comprender y Actuar. Madrid: La Muralla S.A.

Zueck Enríquez, M., Ramírez García, A., Rodríguez Villalobos, J., \& Irigoyen Gutiérrez, H. (2019). Satisfacción en las clases de Educación Física y la intencionalidad de ser activo en niños del nivel de primaria (Satisfaction in the Physical Education classroom and intention to be physically active in Primary school children). Retos, 37, 33-40. https: / /doi.org/10.47197/retos.v37i37.69027

\section{(Footnotes)}

1 Programa desarrollado por la empresa Scientific Software DevelopmentGmbH para el procesamiento de información en investigación cualitativa, el cual tiene licencia de compra por parte del grupo «Mundos Simbólicos: Estudios en Educación y Vida Cotidiana».

${ }^{2}$ Hace referencia a un cálculo aproximado que se realiza mediante la observación sin contar con unos indicadores o criterios claros y se torna muy subjetivo.

${ }^{3}$ Según el documento Orientaciones Pedagógicas para la EF, Recreación y Deporte, las metas de formación son: promover el cultivo personal desde la realidad del ser humano; aportar a la formación del ser humano en el horizonte de su complejidad; contribuir a la construcción de cultura física y a la valoración de sus expresiones autóctonas; apoyar a la formación de cultura ciudadana y valores fundamentales para la convivencia y la paz; apoyar una educación ecológica base de una nueva conciencia sobre el medio ambiente, y por último desarrollar un pedagogía de la inclusión de todas las personas en prácticas de la EF, recreación y deporte. 